\title{
Surgical treatment of trapeziometacarpal joint osteoarthritis
}

\section{GIUSEPPE TACCARDO ${ }^{1}$, ROCCO DE VITIS², GIUSEPPE PARRONE ${ }^{1}$, GIUSEPPE MILANO ${ }^{3}$, FRANCESCO FANFANI ${ }^{1}$}

${ }^{1}$ Orthopaedics and Hand Surgery, Catholic University, Columbus Hospital, Rome, Italy

${ }^{2}$ Orthopaedics and Hand Surgery, Columbus Hospital, Rome, Italy

${ }^{3}$ Department of Orthopedics, Catholic University, "A. Gemelli" Hospital, Rome, Italy

\begin{abstract}
Trapeziometacarpal joint osteoarthritis is a common cause of radial-sided wrist pain that prevalently affects women. It is diagnosed on the basis of a thorough history, physical examination, and radiographic evaluation. While radiographs are used to determine the stage of disease, treatment is dependent on the severity of the symptoms. Non-surgical treatment frequently consists of activity modification, non-steroidal anti-inflammatory drugs, splinting and corticosteroid injections. After failure of conservative treatment, various surgical options exist depending on the stage of the disease. These options range from ligament reconstruction or osteotomy, for early painful laxity, to trapeziectomy, arthrodesis and arthroplasty for more severe osteoarthritis. This article reviews the literature supporting the various surgical treatment options and analyzes the surgical techniques most frequently used in the different disease stages.
\end{abstract}

Key Words: trapeziometacarpal joint, osteoarthritis, rhizoarthrosis, surgical treatment, tendon interposition, ligamentoplasty, arthrodesis.

\section{Introduction}

Trapeziometacarpal joint (TMJ) osteoarthritis (OA), or rhizarthrosis, is a disabling condition of the hand that

\section{Corresponding Author:}

Rocco De Vitis, MD

Orthopaedics and Hand Surgery, Columbus Hospital

Via G. Moscati 31, 00168 Rome, Italy

Tel: +39 3476585278

E-mail: roccodevitis@yahoo.com causes pain, stiffness and weakness, resulting in impaired hand function, strength and dexterity (Fig. 1). It is the second most common degenerative disease of the hand, after distal interphalangeal joint OA (1). About one in four women (30\% of post-menopausal women) and one in 12 men eventually show osteoarthritic changes of the TMJ on radiographs, although the vast majority are asymptomatic (2). The overwhelming prevalence of TMJ OA in females is attributable to different factors: anatomical (a smaller and less congruent shallow saddle joint, flat trapezial facet), hereditary (dysplastic joint surfaces) and hormonal (increased ligament laxity), all resulting in greater joint contact pressure (3). To date there is no longitudinal natural history study suggesting that TMJ OA is caused by certain repetitive work practices. However, a strong association has emerged between excessive joint laxity and the development of premature degenerative joint changes (4). Forrestier (5) was the first to study TMJ OA, and he described it as an isolated pathology. The current view, however, as well expressed by other authors (6-8), is that this condition should be seen as part of a form of arthritic disease that affects several joints. Radiographs of the thumb in three planes and special basal joint stress views are helpful in confirming the diagnosis (4). Further evidence of painful laxity of the TMJ can be obtained from an Eaton and Littler stress radiograph (9). This radiograph, which consists of a postero-anterior $30^{\circ}$ oblique view centered on the thumbs, is taken when the patient's thumb tips are touching and being pressed together. This stresses the TMJ causing radial subluxation. If the TMJ is lax, 50\% of the articular surface of the base of the thumb metacarpal will be seen, on this radiograph, to lie radial to the trapezial articular surface (4). The 

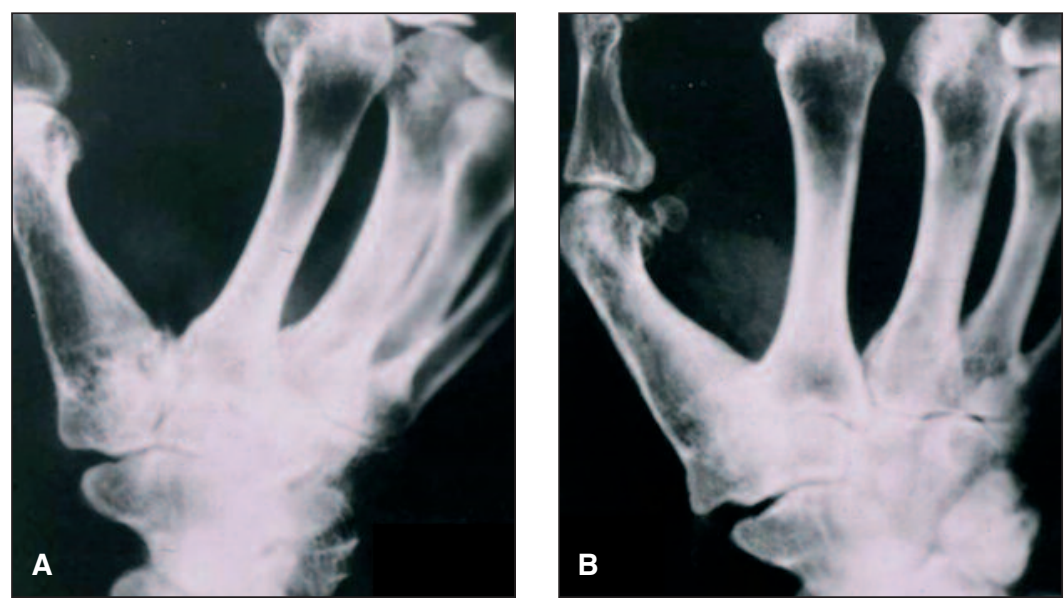

Fig. 1. Radiological follow-up of arthrodesis at two years (A) and five years (B) after surgery.

literature contains several classifications based primarily on evolving radiographic changes. The most widely adopted is that of Dell et al. (10), which divides rhizarthrosis into four stages of evolution, in relation to which the therapy is decided. In stage 1, the treatment used is mainly conservative and symptomatic, involving the use of night splints, rest, orthoses, non-steroidal anti-inflammatory drugs (NSAIDs) and infiltration of corticosteroids. In stage 2, especially in young people and in people involved in heavy manual work, and in stages 3 and 4, arthrodesis was, until 1992, the most widely used treatment; since then most authors have preferred the tendon suspension arthroplasty technique. Finally, in the presence of pan-carpal OA, some authors recently recommended arthroplasty associated with selective denervation of the wrist $(11,12)$.

Of the various procedures available for the treatment of the different stages of OA, it remains uncertain which afford the best outcomes for any given stage of TMJ OA (13). The purpose of this review is to establish whether any one procedure has been shown to be superior to another for a given stage of this disease.

\section{Surgical options}

The TMJ is the upper extremity site most commonly requiring surgical reconstruction for OA (14). Many surgical procedures have been described for this condition, with preferences based mainly on personal experience rather than on a "methodologically sound assessment of primary studies" (15). We have reviewed the use of these many techniques in TMJ OA, highlighting the ones most commonly used in the different disease stages.

\section{Stage I disease}

\section{Ligament reconstruction}

Stabilization of the TMJ by isolated reconstruction of the palmar beak ligament (deep anterior oblique ligament) should be performed only in stage I disease (4,16-18). However, for long-term success, the articular surfaces must be eburnation-free, a state that can be best ascertained intraoperatively. Gerwin et al. (16) carried out a prospective, randomized study. In Group I (nine patients), ligament reconstruction was performed to suspend the first metacarpal as well as place a rolled tendon interposition to fill the void created by resection of the trapezium. In Group II (11 patients), ligament reconstruction alone was performed, with the use of a suture anchor. There was no difference between the two groups in range of motion of the thumb, grip strength, lateral pinch strength, the ability to perform activities of daily living, or subjective satisfaction with the procedures.

The most commonly used ligament reconstruction technique is the one described by Eaton and Littler (9) that uses $50 \%$ of the width of the flexor carpi radialis (FCR) tendon. These authors assessed 18 patients who underwent a ligament reconstruction procedure for painful, unstable thumb carpometacarpal joints. Reconstruction was performed only when splinting and anti-inflammatory regimens had failed. The results were directly related to the extent of articular degeneration. Restoration of strength, mobility, and freedom from pain were better if surgery was performed before significant articular damage was noted on roentgenograms.

\section{Metacarpal extension osteotomy}

Extension osteotomy, described by Wilson in 1973 (19), has been shown to shift contact stresses dorsally and may give symptomatic relief in stage I or early 
stage II disease, but not in advanced stage III and in stage IV OA. The rationale of this procedure is to compensate for the incompetence of the beak ligament, which increases the volar shear stresses and leads to cartilage breakdown volarly $(20,21)$.

The technique has been reported by several authors (2023). In a review of 41 thumbs at a mean of seven years, Hobby et al. (22) found good or excellent pain relief in $80 \%$. The procedure has been proposed as best suited to young individuals with high demands, as it is a more durable procedure than arthroplasty and results in less restriction of motion than arthrodesis (4). However, it is not widely used and, also for this reason, its outcome has never been compared with that of other procedures.

\section{Stage II and III disease}

\section{Excisional arthroplasty (trapeziectomy)}

Gervis first described trapezial excision in 1949 (24). This method may be appropriate for cases of advanced stage II to stage IV disease or in low-demand older patients (4). All procedures that involve excision of the trapezium can be performed both in patients with isolated TMJ OA and in those whose TMJ OA is combined with scapho-trapezial-trapezoidal (STT) joint OA (24).

The technique has been reported by several authors (2428). In a five-year follow-up assessment of 34 simple trapezial excisions, Varley et al. (25) reported that only $47 \%$ were completely pain-free. Davis et al. (26) randomized 183 thumbs with TMJ OA to treatment by simple trapeziectomy, trapeziectomy with palmaris longus interposition, or trapeziectomy with ligament reconstruction and tendon interposition. A K-wire was passed across the trapezial void during each of the 183 surgeries to hold the base of the thumb metacarpal at the level of the index carpometacarpal joint and was retained for four weeks in each case. All the patients wore a thumb splint for six weeks. Each patient underwent subjective and objective assessments of thumb pain, stiffness and strength before surgery and at three months and one year after surgery. The outcomes of the three groups were very similar at the one-year follow-up evaluation. Therefore, in the short term at least, trapeziectomy appears to offer no benefit over tendon interposition or ligament reconstruction.
Trapeziectomy and interpositional arthroplasty

Froimson (29) first described the technique involving the insertion of a rolled-up piece of FCR (serving as a "spacer") into the cavity left after trapeziectomy, in the absence of ligament reconstruction.

This technique was then adapted, using the abductor pollicis longus (APL) tendon, lyophilized homologous dura mater and fascia lata as interpositional material. Although these procedures initially appeared to considerably decrease thumb shortening, it was subsequently noted that, over time, thumb length was lost due to the metacarpal settling into the interpositional material, and that no significant improvement in function was actually obtained (4). Raven et al. (30) compared three surgical procedures in 63 patients (74 thumbs): resection arthroplasty (18 thumbs), trapeziectomy with tendon interposition (17 thumbs) and trapeziometacarpal arthrodesis (28 thumbs). They found no difference between the three groups in grip and tip pinch strength or in pain on palpation. None of their trapeziectomy patients needed a re-operation, one patient in the resection arthroplasty group had a re-operation, and 22 patients in the arthrodesis group had one or more reoperations for hardware removal or because of a complication.

\section{Trapeziectomy combined with ligament reconstruction} and tendon interposition

For disease limited to the TMJ, the most commonly used procedure is the ligament reconstruction and tendon interposition (LRTI) arthroplasty described by Burton and Pellegrini in 1986 (31), in which half of the width of the FCR tendon is used to support the base of the thumb metacarpal after excision of the trapezium. These authors found that LRTI arthroplasty more consistently improved pinch strength, increased grip strength endurance, and restored thumb web space than did silicone implant arthroplasty. Proximal metacarpal migration averaged only $11 \%$ of the initial arthroplasty space versus nearly $50 \%$ loss of height with silicone implants. Subluxation averaged only $7 \%$ of the width of the thumb metacarpal base relative to the scaphoid versus subluxation of $35 \%$ of the base of the implant with silicone arthroplasty. Excellent results were achieved in 23 thumbs or $92 \%$ of cases. No deterioration of function or stability was noted over time, and no revision procedures were necessary (31).

This technique has also been reported by other authors (32-36). Tomaino et al. (35,36) described an average 
nine-year follow-up of 24 thumbs in 22 patients. In their opinion, LRTI arthroplasty provided a stable and functional reconstruction of the thumb, resulting in excellent relief of pain and a significant increase in strength for as long as 11 years after the procedure.

\section{Partial trapeziectomy}

An alternative to LRTI after trapeziectomy is resection of only part of the trapezium and interposition of autologous or other tissue $(4,37-39)$. This technique makes it possible to exploit the remaining trapezium as a spacer and buttress and to address only the arthritic joint surface. Theoretically, this would improve stability and thumb strength and lead to less proximal migration of the thumb ray (40). This type of arthroplasty is appropriate for stage II and III disease. Noland et al. (37) evaluated 13 patients (16 thumbs) who underwent partial trapeziectomy between 1995 and 2005. They found that partial trapeziectomy for TMJ OA provided long-lasting relief of symptoms in patients with radiographically abnormal but clinically insignificant scaphotrapezial joint degeneration.

\section{Prosthetic TMJ arthroplasty}

Several authors have reported the use of TMJ prostheses $(41-46)$. The aim of the procedure, besides eliminating painful articulating surfaces, is to establish a stable force column during pinching and gripping (4). Prosthetic replacement of the TMJ may be divided into three broad categories: (a) arthroplasties that involve resurfacing of either the trapezium or the metacarpal base, for TMJ OA only; (b) trapezium replacement arthroplasties for patients with pantrapezial OA, and (c) total joint replacement. O'Leary et al. (47) reviewed 23 patients who had undergone trapeziectomy and silicone rubber ball interposition for TMJ OA. They found that, of the 23 patients, two had pain at rest and four had some discomfort on exertion. Mean post-operative thumb extension was 37 degrees, whilst mean palmar abduction was 40 degrees. Mean post-operative grip strength was $19 \mathrm{~kg}$ and thumb-pinch strength was $4.0 \mathrm{~kg}, 77 \%$ and $78 \%$ of the age- and sex-matched normal values, respectively. There were no cases of prosthetic dislocation, prosthetic fracture or silicone synovitis.

Tagil et al. (41) operated on 26 patients. After excision of the trapezium, they randomized the patients to receive either a Swanson silicone trapezium implant or a tendon interposition arthroplasty with APL. They found no infection or clinical silicone synovitis, and no major radiographic cyst formation. Two endoprostheses dislocated early. All 13 patients in the Swanson group and 11 of the 13 in the APL group were subjectively satisfied at two-to-five-year follow-up. All the patients were free of pain at rest and on light exertion, but half of the patients in each group still experienced pain with heavy work. Thumb pinch strength and range of motion were not significantly different in the two groups. The trapezial space decreased both with load and length of follow-up in both groups. The authors concluded that both methods gave good, but not complete, pain relief and neither produced better results, in the short term, than those reported with other procedures.

\section{Arthrodesis}

Arthrodesis (Fig. 2) of the TMJ has traditionally been the procedure chosen for younger, active people (often with a post-traumatic etiology) who have high joint demands, requiring a strong grip and pinch because of their occupation (4). It is indicated for people with stage III and IV OA. While it provides stability, strength and pain relief, it does so at the expense of mobility and transfer of joint reaction forces to other joints (48).

The literature contains several reports on the use of TMJ arthrodesis (49-53). The most widely used technique is the traditional one described by Muller in 1949 (54), which is performed under regional anesthesia and limb ischemia. Cavallazzi et al. (55) carried out a 10-year follow-up of 42 patients (a total of 43 hands) submitted to arthrodesis of the TMJ for dege-

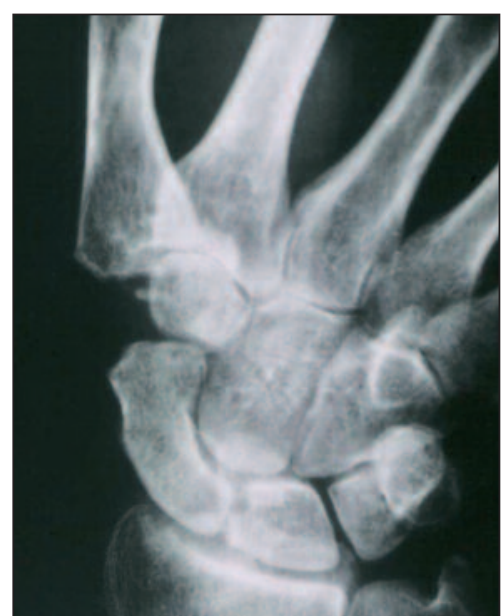

Fig. 2. Radiological long-term follow-up of suspension arthtoplasty according to Weilby and Ceruso et al. 
nerative OA. These patients obtained relief of pain, maintenance of stability and mobility of the first ray, and good function of the operated hands, and were pleased with the results. Catalano et al. (56) described the clinical and radiological findings in 40 cases of TMJ OA treated surgically. Twenty patients had undergone arthrodesis and 20 had undergone tendon arthroplasty. The two techniques provided very good results that were largely overlapping (except that arthroplasty was associated with increased mobility and arthrodesis with greater plier strength). The authors concluded that the presence of concomitant peri-trapezial OA constitutes a mandatory indication for arthroplasty. Arthrodesis, on the other hand, is more indicated in the presence of a severe deformity in adduction of the first metacarpal, sometimes incompletely corrected by simple arthroplasty.

Kenniston and Bozentka (52) found that arthrodesis was a viable option for isolated TMJ OA, making it possible to create a pain-free, strong and stable thumb.

\section{Arthroplasty with tenoplasty in suspension}

The most commonly used tendon arthroplasty (Figs. 3-5) procedure is the one described by Ceruso et al. (57), who took up and modified Weilby's original idea (58). The technique has also been reported by other authors $(59,60)$.

According to Weilby (58), after removal of the trapezium, imbrication of the APL and fixation of the latter with a strip of FCR tendon, a stable arthroplasty is obtained with three quarters of normal mobility and half to full power. Of the first 100 operated thumbs, only 15 patients had slight pain on heavy use; the others were pain-free. Three patients had radial nerve lesions with minor symptoms. Seven patients developed de Quervain's syndrome and two had rupture of the APL. These complications have since been avoided by routinely splinting the first extensor compartment. Four cases with unstable joints required further surgery, and one joint became stiff due to extra-articular conditions (58).

\section{Stage IV disease}

In the presence of stage IV disease, possible procedures include arthrodesis, trapeziectomy, trapeziectomy with LRTI, interpositional arthroplasties and TM and STT joint replacements (all of which we have already dealt with). There are no significant differences between them.

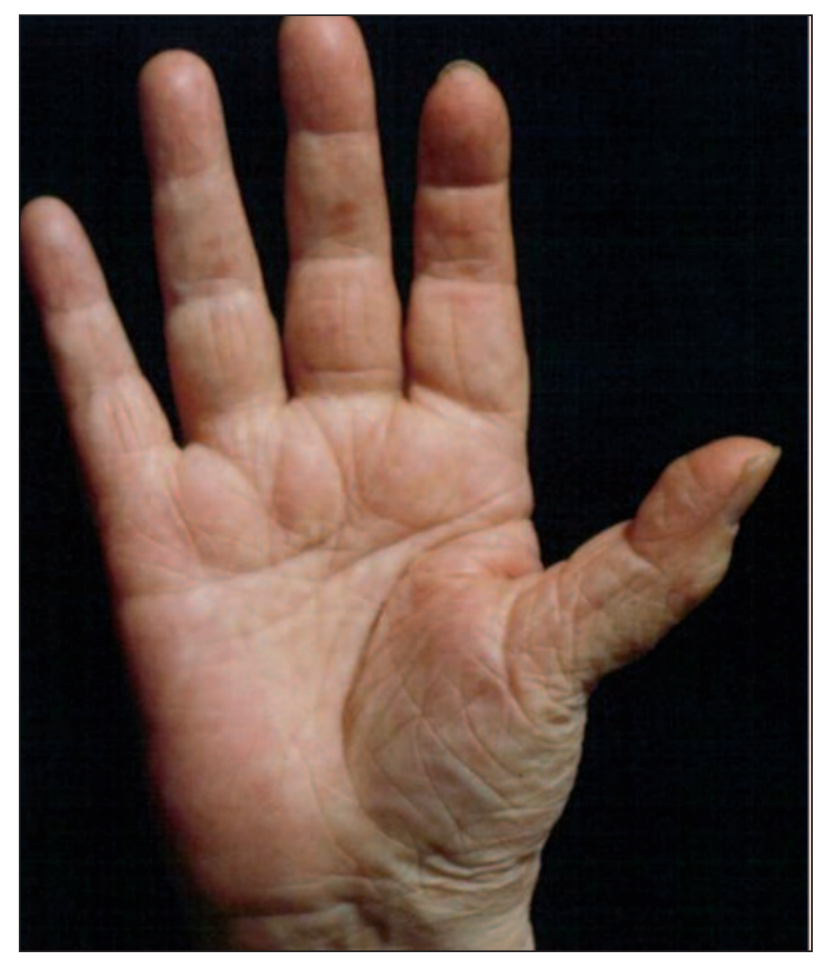

Fig. 3. Deformity typical of advanced TMJ OA.

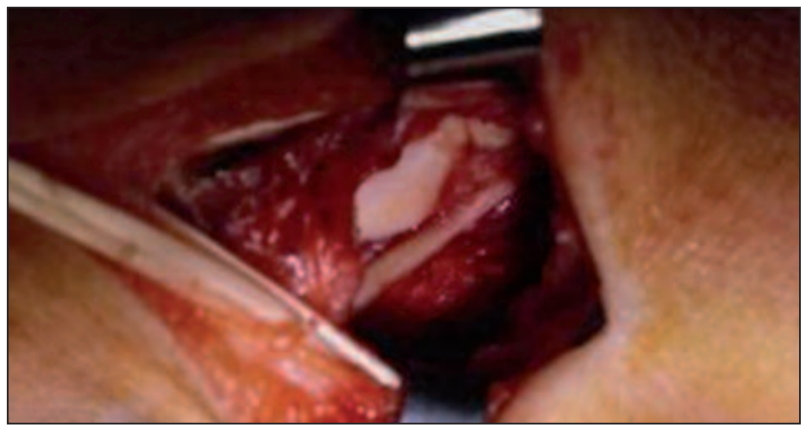

Fig. 4. Intraoperative view of a trapeziectomy.

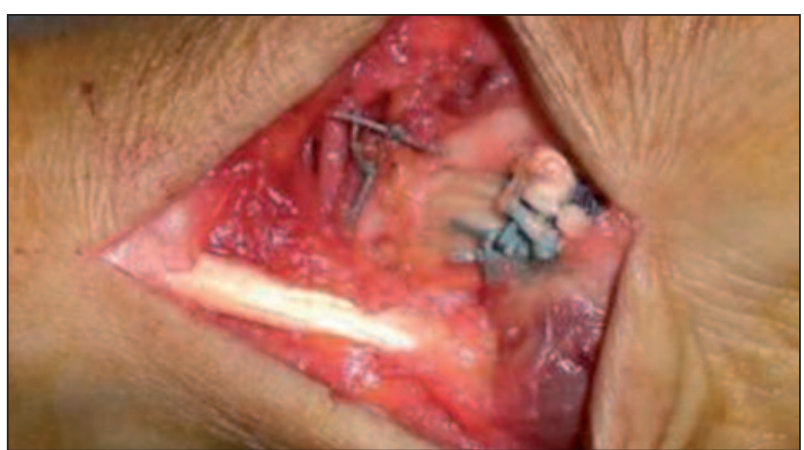

Fig. 5. Intraoperative view of a suspension arthroplasty according to Weilby and Ceruso et al. 


\section{Conclusions}

Of all the techniques considered, the ones most used, and for which there exist retrospective studies evaluating their long-term effectiveness, are arthrodesis and arthroplasty (4). The advantages of arthrodesis are that it reduces the painful symptoms and ensures recovery of the stability of the base of the thumb and of gripping force, thereby leaving the individual the possibility of performing heavy work. However, it requires immobilization in plaster followed by a period of rehabilitation for complete recovery of function. It also leads to limitation of the mobility of the thumb, especially in retropulsion, and thus to a decrease in manual dexterity. Conversely, tendon arthroplasty guarantees excellent mobility and function of the thumb, including the possibility of performing precise and delicate movements. This method requires a shorter immobilization, which normally implies an earlier functional recovery. However, while it eliminates the painful arthritic symptomatology, it can be associated, in some cases, with post-operative pain. Furthermore, arthroplasty is a more complex technique that, compared with arthrodesis, gives lower overall results in terms of residual plier strength. For all these reasons, the use of either technique should be decided on the basis of the patient's age and type of work, and on the features (clinical and radiological) of the arthritic process. In the presence of concomitant peri-trapezial OA, arthroplasty is generally recommended (56).

Despite these considerations, no procedure has yet demonstrated any superiority over another in terms of pain relief, physical function, global patient assessment, or range of motion (61). Trapeziectomy has fewer complications than trapeziectomy with LRTI. Therefore, the current evidence, failing to establish the superiority of any surgical procedure for any given stage of TMJ OA, does not provide a basis for specific treatment recommendations (61).

\section{References}

1. Li YK, White C, Ignacy TA, Thoma A. Comparison of trapeziectomy and trapeziectomy with ligament reconstruction and tendon interposition: a systematic literature review. Plast Reconstr Surg 2011;128:199-207.

2. Armstrong AL, Hunter JB, Davis TR. The prevalence of degenerative arthritis of the base of the thumb in post-menopausal women. J Hand Surg Br 1994;19:340-341.

3. Katarincic JA. Thumb kinematics and their relevance to function. Hand Clin 2001;17:169-174.

4. Batra S, Kanvinde R. Osteoarthritis of the thumb trapezio- metacarpal joint. Current Orthopaedics 2007,21:135-144.

5. Forrestier J. Losteo-arthrite seche trapezio-metacarpienne. Presse Med 1937;45:315-317.

6. Allieu Y, Pequignot JP, Asencio G, Gomis R, Bahri H, Escare P. Swanson trapezial implant in the treatment of peritrapezial arthrosis. A study of eighty cases. Ann Chir Main 1994;3:113-123.

7. Saffar P. La rhizarthrose. Paris: Monographie du G.E.M. Eds Ex Scientif Française 1990.

8. Swanson AB. Disabling arthritis at the base of the thumb: treatment by resection of the trapezium and flexible (silicone) implant arthroplasty. J Bone Joint Surg Am 1972;54:456-471.

9. Eaton R, Littler J. Ligament reconstruction for the painful thumb carpometacarpal joint. J Bone Joint Surg Am 1973;55:1655-1666.

10. Dell PC, Brushart TM, Smith RJ. Treatment of trapeziometacarpal arthritis: results of resection arthroplasty. J Hand Surg Am 1978;3:243-249.

11. Abbiati G, Del Bene M, Petrolati M, Ciapparelli A, Amadei F. Surgical treatment of trapezio-metacarpal arthritis: arthrodesis vs tendon arthroplasty. G.I.O.T. 2000;27:134-139.

12. Loréa P, Dury M, Marin Braun F, Dekkaï T, De Mey A, Foucher G. Trapeziometacarpal denervation. Description of surgical technique and preliminary results from a prospective series of 14 cases. Chir Main 2002;21:209-217.

13. Hartigan BJ, Stern PJ, Kiefhaber TR. Thumb carpometacarpal osteoarthritis: arthrodesis compared with ligament reconstruction and tendon interposition. J Bone Joint Surg Am 2001;83-A:1470-1478.

14. Pellegrini VD, Olcott CW, Hollenberg G. Contact patterns in the trapeziometacarpal joint: the role of the palmar beak ligament. J Hand Surg Am 1993;18:238-244.

15. Martou G, Veltri K, Thoma A. Surgical treatment of osteoarthritis of the carpometacarpal joint of the thumb: a systematic review. Plast Reconstr Surg 2004;114:421-432.

16. Gerwin M, Griffith A, Weiland A, Hotchkiss R, McCormack R. Ligament reconstruction basal joint arthroplasty without tendon interposition. Clin Orthop Relat Res 1997;(342):42-45.

17. Kriegs-Au G, Petje G, Fojtl E, Ganger R, Zachs I. Ligament reconstruction with or without tendon interposition to treat primary thumb carpometacarpal osteoarthritis. Surgical technique. J Bone Joint Surg Am 2005;87 Suppl 1:78-85.

18. Adams BD, Unsell RS, McLaughlin P, Niebauer trapeziometacarpal arthroplasty. J Hand Surg Am 1990;15:487-492.

19. Wilson J. Basal osteotomy of the first metacarpal in treatment of arthritis of the carpometacarpal joint of the thumb. Br J Surg 1973;60:854-858.

20. Pellegrini VD Jr. Osteoarthritis of the trapeziometacarpal joint: the pathophysiology of articular cartilage degeneration. Anatomy and pathology of the aging joint. J Hand Surg Am 1991;16:967-974.

21. Poole JU, Pellegrini VD Jr. Arthritis of the thumb basal joint complex. J Hand Ther 2000;13:91-107.

22. Hobby JL, Lyall HA, Meggitt BF. First metacarpal osteotomy for trapeziometacarpal osteoarthritis. J Bone Joint Surg Br 1998;80:508-512.

23. Holmberg J, Lundborg G. Osteotomy of the first metacarpal for osteoarthrosis of the basal joints of the thumb. Scand J Plast Reconstr Surg Hand Surg 1996;30:67-70.

24. Gervis WH. Excision of the trapezium for osteoarthritis of the trapezio-metacarpal joint. J Bone Joint Surg Br 1949; 31:537-553.

25. Varley GW, Calvey J, Hunter JB, Barton NJ, Davis TR. Excision of the trapezium for osteoarthritis at the base of the thumb. J Bone Joint Surg Br 1994;76:964-968. 


\section{G. Taccardo et al.}

26. Davis TR, Brady O, Barton NJ, Lunn PG, Burke FD. Trapeziectomy alone, with tendon interposition or with ligament reconstruction? J Hand Surg Br 1997,22:689-694.

27. Dhar S, Gray IC, Jones WA, Beddow FH. Simple excision of the trapezium for osteoarthritis of the carpometacarpal joint of the thumb. J Hand Surg Br 1994,19:485-488.

28. Vandenbroucke J, De Schrijver F, De Smet L, Fabry G. Simple trapeziectomy for treatment of trapeziometacarpal osteoarthritis of the thumb. Clin Rheumatol 1997,16:239-242.

29. Froimson AI. Tendon arthroplasty of the trapeziometacarpal joint. Clin Orthop Relat Res 1970,70:191-199.

30. Raven EE, Kerkhoffs GM, Rutten S, Marsman AJ, Marti RK, Albers GH. Long term results of surgical intervention for osteoarthritis of the trapeziometacarpal joint: comparison of resection arthroplasty, trapeziectomy with tendon interposition and trapezio-metacarpal arthrodesis. Int Orthop 2007,31:547-554.

31. Pellegrini VD Jr, Burton RI. Surgical management of basal joint arthritis of the thumb. Part 1. Long-term results of silicone implant arthroplasty. J Hand Surg Am 1986,11:309-324.

32. Belcher HJ, Nicholl JE. A comparison of trapeziectomy with and without ligament reconstruction and tendon interposition. J Hand Surg Br 2000,25:350-356.

33. Davis TR, Brady O, Dias JJ. Excision of the trapezium for osteoarthritis of the trapeziometacarpal joint: a study of the benefit of ligament reconstruction or tendon interposition. J Hand Surg Am 2004,29:1069-1077.

34. Lins RE, Gelberman RH, Mc Keown L, Katz JN, Kadiyala RK. Basal joint arthritis: trapeziectomy with ligament reconstruction and tendon interposition arthroplasty. J Hand Surg Am 1996,21:202-209.

35. Tomaino MM, Pellegrini VD, Burton RI. Arthroplasty of the basal joint of the thumb. Long-term follow-up after ligament reconstruction with tendon interposition. J Bone Joint Surg Am 1995,77:346-355.

36. Tomaino MM. Ligament reconstruction tendon interposition arthroplasty for basal joint arthritis. Rationale, current technique, and clinical outcome. Hand Clin 2001,17:207-221.

37. Noland SS, Saber S, Endress R, Hentz VR. The scaphotrapezial joint after partial trapeziectomy for trapeziometacarpal joint arthritis: long-term follow-up. J Hand Surg Am 2012,37:1125-1129.

38. Cristiani G, Marcuzzi A, Marcialis M, Castagnini L, Caroli A. Partial trapeziectomy, ligament reconstruction and interpositional arthroplasty for trapeziometacarpal arthritis of the thumb. J Hand Surg Br 1997,22(Suppl 1):30.

39. Phaltankar PM, Magnussen PA. Hemiarthroplasty for trapeziometacarpal arthritis - a useful alternative? J Hand Surg Br 2003,28:80-85.

40. Tropet Y, Gallinet D, Lepage D, Gasse N, Obert L. Treatment of trapeziometacarpal osteoarthritis by partial trapeziectomy and costal cartilage autograft. A review of 100 cases. Chir Main 2012,31:145-151.

41. Tagil M, Kopylov P. Swanson versus APL arthroplasty in the treatment of osteoarthritis of the trapeziometacarpal joint: a randomized study in 26 patients. J Hand Surg Br 2002,27: 452-456.
42. Braun RM. Total joint replacement at the base of the thumb - preliminary report. J Hand Surg Am 1982,7:245-251.

43. Chakrabarti AJ, Robinson AH, Gallagher P. De la Caffinière thumb carpometacarpal replacements. 93 cases at 6 to 16 years follow-up. J Hand Surg Br 1997,22:695-698.

44. Hofammann DY, Ferlic DC, Clayton ML. Arthroplasty of the basal joint of the thumb using a silicone prosthesis. Longterm follow-up. J Bone Joint Surg Am 1987,69:993-997.

45. Nakajima H, Masada K, Ohno H, Shinmei M. A new arthroplasty of the basal joint of the thumb using a silicone ball. Journal of Orthopaedic Surgery 1996,4:61-66.

46. Swanson AB, Swanson GD. Osteoarthritis in the hand. J Hand Surg Am 1983,8:669-675.

47. O' Leary ST, Grobbelaar AO, Goldsmith N, Smith PJ, Harrison DH. Silicone arthroplasty for trapeziometacarpal arthritis. J Hand Surg Br 2002,27:457-461.

48. García-Paredero E, Cecilia-López D, Suárez-Arias L, ResinesErasun C. Results of surgical treatment rhizarthrosis by means of trapeziometacarpal joint arthrodesis with a quadrangular osteosynthesis plate. Rev Esp Cir Ortop Traumatol 2010,54:203-207.

49. Carroll RE. Arthrodesis of the carpometacarpal joint of the thumb. A review of patients with a long postoperative period. Clin Orthop Relat Res 1987, (220):106-110.

50. Galli S, Megaro A, Filippo GD, Codato F, Pazzaglia U. Arthrodesis versus suspension arthroplasty in the treatment of carpometacarpal arthritis of the thumb. J Hand Surg Br 2002,27(Suppl 1):26.

51. Karlsson MK. Arthrodesis of the trapeziometacarpal joint. Scand J Plast Reconstr Surg Hand Surg 1990,25:167-171.

52. Kenniston JA, Bozentka DJ. Treatment of advanced carpometacarpal joint disease: arthrodesis. Hand Clin 2008,24:285-294.

53. Schröder J, Kerkhoffs GM, Voerman HJ, Marti RK. Surgical treatment of basal joint disease of the thumb: comparison between resection-interposition arthroplasty and trapezio-metacarpal arthrodesis. Arch Orthop Trauma Surg 2002,122:35-38.

54. Muller GM. Arthrodesis of the trapezio-metacarpal joint for osteoarthritis. J Bone Joint Surg Br 1949,31B:540-542.

55. Cavallazzi RM, Spreafico G. Trapezio-metacarpal arthrodesis today: why? J Hand Surg Br 1986,11:250-254.

56. Catalano F, Fanfani F, Taccardo G, Pagliei A. Artrodesi ed artroplastica a confronto nel trattamento della rizoartrosi. Riv Chir Mano 1988,25:255-259.

57. Ceruso M, Innocenti M, Angeloni R, Lauri G, Bufalini C. Lartrosi del primo raggio digitale. Riv Chir Mano 1991,28:67-75.

58. Weilby A. Tendon interposition arthroplasty of the first carpo-metacarpal joint. J Hand Surg Br 1988;13:421-424.

59. Herren D, Simmen B. The resection-suspension interposition-arthroplasty for the treatment of peritrapezial arthritis. J Hand Surg Br 1997,22(Suppl 1):29.

60. Robinson D, Aghasi M, Halperin N. Abductor pollicis longus tendon arthroplasty of the trapezio-metacarpal joint: surgical technique and results. J Hand Surg Am 1991,16:504509.

61. Wajon A, Carr E, Edmunds I, Ada L. Surgery for thumb (trapeziometacarpal joint) osteoarthritis. Cochrane Database Syst $\operatorname{Rev} 2009$, 7:CD004631. 